\title{
Evaluación del desarrollo de competencias transversales y destrezas en el manejo del modelo e-learning en programas de pregrado en Latinoamérica
}

\author{
Evaluation of the Development of Transveral Competences and Skills for \\ Management of e-learning Model in Undergraduate Programs in Latin \\ America
}

\author{
Juan Fernando Ossa Stipcianos \\ Corporación Universitaria del Meta. Colombia \\ juan.ossa@unimeta.edu.co
}

\begin{abstract}
Resumen
La implementación del modelo de enseñanza e-learning en Latinoamérica se encuentra en etapa de crecimiento, cumpliendo con los parámetros de calidad en relación con el desarrollo de competencias trasversales y especificas. El presente artículo muestra el resultado del análisis del desarrollo de competencias transversales (cognitivas, socio afectivas, comunicativas) y de destrezas en el manejo del hardware y software (experticia) en la implementación del modelo de enseñanza e-learning en un programa profesional de pregrado. La metodología utilizada para este estudio fue mixta, utilizando investigación cualitativa para describir actitudes, comportamientos y motivaciones de los estudiantes frente al uso del modelo e-learning e investigación cuantitativa para medir el nivel de destreza en el uso del modelo por los estudiantes. Los resultados revelan que el éxito en el desarrollo de las competencias descritas en la utilización del modelo e-learning depende del nivel de habilidad que adquieran los estudiantes y docentes, como también de la iniciativa del docente en el diseño instruccional y del seguimiento del desempeño de sus estudiantes.
\end{abstract}

\section{Palabras clave}

Competencias transversales, E-learning, Destrezas, Pregrado

\begin{abstract}
Implementation of the e-learning model in Latin America is at a growing stage, meeting the quality parameters in relation to transversal and specific competences development. This article shows the results of an analysis of the development of transversal competences (cognitive, socio affective, and communication competences) and skills in managing hardware and software for the implementation of e-learning in a professional undergraduate program. The methodology used for this study was a mixed one; qualitative research to describe attitudes, behaviors and motivations of the students in the use of the e-learning model, and quantitative research to measure the level of expertise in the use of the model by students. The results reveal that success in the development of the described competences in the use of the e-learning model depends on the level of expertise they students and teachers acquire, as wel as on the initiative of teachers in instructional design and monitoring of the performance of their students.
\end{abstract}

\section{Key words}

Generic competences, E-learning, Skills, Undergraduate 


\section{Introducción}

La evolución de la educación se ha desarrollado sistemáticamente en relación con la evolución de la sociedad en tres diferentes entornos: natural, urbano y telemático, en cada uno de estos entornos se han creado diferentes tipos de condiciones sociales, culturales y ambientales. Desde los inicios de la historia hasta 1960 los entornos eran Naturales y Urbanos para el desarrollo de las sociedades. En los años 50`s, 60`s, 70`s y principios de los 80’s el uso del computador era exclusivo de las grandes corporaciones y los medios de comunicación más utilizados era el teléfono, la televisión y los medios escritos. En los años 80`s, 90`s y 00, el computador pasó a ser un instrumento común en los hogares y el desarrollo de las comunicaciones se dio de forma vertiginosa con la telefonía móvil y el uso masificado de Internet. El entorno que se desarrolló fue el telemático en la sociedad donde primaba la información de tipo sincrónica en la educación presencial y asincrónica en la educación a distancia no era necesaria la presencia de los docentes y los estudiantes en el mismo tiempo y en el mismo espacio. Las TIC ayudaron a acortar las distancias y romper las barreras del tiempo facilitando el uso de la información, es así como se puede acceder por medio de las TIC a cualquier fuente de información en cualquier lugar. Pasamos de una sociedad de la información a una sociedad del conocimiento, esto se debe al desarrollo tecnológico, que ha mejorado exponencialmente con el uso de los medios de información y comunicación (Echeverría, 1999).

El impacto de las TIC en la educación ha sido importante, pero no se ha desarrollado en todo su potencial, en la mayoría de los países de Europa se ha implementado de forma desigual. La tecnología ha creado diferentes y variados modelos de enseñanzaaprendizaje en la era de la sociedad del conocimiento: el multimedia, el informático, el basado en internet, hasta el modelo e-learning, mobile-learning y blenden-learning (García, 2007). El modelo e-learnig es el uso de nuevas tecnologías en multimedia e internet que mejoran la calidad del aprendizaje facilitando el acceso a los recursos y los servicios, como también intercambios remotos y colaboraciones en campos muy diversos.(Sarmento y Durão, 2009).

En los países en desarrollo, la implementación del modelo e-learning no tiene problemas en cuanto a la infraestructura con relación a la disponibilidad de computadores. Pero hay debilidades en relación a la educación, al acceso de bibliotecas confiables, a suficiente contenido internacional y local, y a una cultura política que tenga intención de apoyar el aprendizaje virtual. Es necesario que los gobiernos garanticen un acceso a internet de forma barata y confiable, y unos docentes que estén preparados para diseñar y compartir contenidos con estudiantes dispuestos a aprender (Eduteka, 2009).

En la década de los noventa en América Latina tuvo su origen la mayor parte de los programas a distancia virtual; estos surgieron por necesidad ofertar un modelo de educación transnacional de oferta libre (Toffler, 2006) citado por (Estévez, J. A., Castro-Martínez, J., \& Granobles, H. R.,2015). En el año 2000 existían 164.000 
estudiantes matriculados que equivale al 1,3\% del total de la matrícula, en el año 2006 este porcentaje subió al 5\%, (Ieslac, 2006) y en el 2012 se reportó 1,5 millones de estudiantes matriculados que corresponde al 7,5\% de la matrícula (Rama, 2013).

Los países líderes en educación a distancia privada en América Latina son México, Brasil, Ecuador, Perú, República Dominicana y Argentina; en el sector público son líderes Costa Rica, Venezuela, Honduras, Colombia y Uruguay. En México, Venezuela y Colombia hay procesos de enseñanza 100\% virtuales (Rama, 2013). La plataforma de e-learning más utilizada es Moodle con un porcentaje de uso del 32,6\% le sigue WebCT con un 17\% y Blachboard con un 15,1\% Claroline 11,7\%. (Garcés Arguello, E. R., \& Rivera, C. J., 2006). Los ingresos provenientes del mercado de e-learning en América Latina fueron de 1.400 millones de dólares en 2013. Se prevé para el 2016 que el sector tendrá 2.300 millones de dólares de facturación. Los países con mayor crecimiento serán Brasil con una expansión del 21,5 \%, Colombia con un 18,6 \%, Bolivia con un $17,8 \%$ y Chile con un $14,4 \%$ (Obs-edu.com, 2014).

Los principales desafíos y tendencias para el 2016 en relación al incremento de $e$ learning en América Latina son el desarrollo del aprendizaje adaptativo o formación personalizada, la consolidación del mobile-learning y la gamificación (uso de herramientas, diseños y aplicaciones para desarrollar juegos y videojuegos pero aplicados a la educación) en los procesos formativos, la evolución a la utilización de blended learning y los Moocs (massive online open courses o cursos online masivos y abiertos), la utilización de los repositorios digitales y la importancia de las redes sociales como responsables de la mejora de los canales de comunicación y capacitación (Americalearningmedia.com, 2016).

La educación a distancia en Colombia tuvo su aparición en 1941 con la Radiodifusora Nacional, en 1957 se da origen a la televisión educativa, luego se reglamenta mediante el decreto 1820 de 1983 la educación abierta y a distancia sustentando el origen en 1982 de la Universidad Nacional Abierta y a Distancia (Estévez, J. A., Castro-Martínez, J., \& Granobles, H. R., 2015). En los años 90 las tics irrumpen el país dando origen a nuevas formas de comunicaciones, conectividad y computación determinante para el desarrollo de la educación a distancia virtual, a finales de los noventas internet era el principal acceso a esta modalidad de educación pero su cobertura era del $1 \%$ de la población colombiana y la disponibilidad de computadores era incipiente (Facundo, 2002).

En 1992 se inicia la educación a distancia virtual cuando el Instituto Tecnológico de Monterrey en convenio con la Universidad Autónoma de Bucaramanga ofrecieron programas a distancia (maestrías) que fueron ofertadas por el convenio de la Red Mutis en 1995/96 mediante clases satelitales realizadas en México. En 1998 se inicia el año de la virtualidad en Colombia debido al ofrecimiento de programas propios de pre-grado por la universidad Militar Nueva Granada y Universidad Católica del Norte pionera como universidad totalmente virtual (Facundo, 2002).

Evaluación del desarrollo de competencias transversales y destrezas en el manejo del modelo e-learning en programas de pregrado en Latinoamérica. Juan Fernando Ossa Stipcianos.

Página 3 de 28 
En el 2011 existían 266 programas ofrecidos por las Instituciones de Educación Superior en modalidad virtual, 563 programas en modalidad de distancia tradicional y 10.002 programas en modalidad presencial (Alvarado, Calderón, 2013) citado por (Estévez, J. A., Castro-Martínez, J., \& Granobles, H. R., 2015). En 2013 el número de pregrados y posgrados virtuales apenas es el 2,5\% de la oferta de programas activos; y en programas de distancia llega al 9\%. La tendencia estadística muestra que los programas virtuales duplicarán los programas de distancia tradicional teniendo en cuenta el aumento de la conectividad de Internet en muchas regiones del país (Martínez, 2013). Es importante el interés de las instituciones gubernamentales en fomentar la educación virtual en Colombia. (Suárez, E. J. C., Salinas, E. R, 2009). La educación a distancia y virtual está crecimiento en Colombia por medio del ofrecimiento de un amplia oferta de educación superior en diversas formas y expresiones (Anton, J., Rincón Prado, J. 2013).

La madurez digital (e-maturity) se presenta de forma diferente entre países, en donde algunas de las escuelas han incorporado las TIC dentro del currículo, demostrando un alto nivel de uso en procesos de enseñanza y aprendizaje en diferentes asignaturas y áreas. Pero en la mayoría de las instituciones educativas se encuentra en su etapa inicial con poco desarrollo de aprendizaje virtual (e-learning) y con pocas mejoras en enseñanza y aprendizaje (Eduteka, 2009). La destreza en el manejo las Tics por parte de los estudiantes y docentes es importante para el desarrollo del modelo e-learning. Esta destreza se describe según Eduteka (2007) con el término de EXPERTICIA como una serie de nuevas competencias en el manejo de la información y en la construcción del conocimiento; competencias relacionadas con en el manejo de hardware y software; competencias en la búsqueda de contenidos de información, y en el manejo de las comunicaciones y capacidades intelectuales de orden superior (Eduteka, 2007).

Según García (2007): "la educación ha sido un factor clave para integrar a las personas en los nuevos modelos de sociedad, facilitando la adquisición de nuevas habilidades y competencias".(p.18). La educación se ha desarrollado según las condiciones de cada uno de los entornos mencionados propiciando el desarrollo de los individuos de forma integral. La educación tiene el reto de formar en cada entorno natural, urbano y telemático atendiendo a las necesidades con estructuras y medios diferentes (García, 2007), como también de guiar a los estudiantes en el manejo de la información para el desarrollo del conocimiento mediante procesos de aprendizaje cognitivos que ayuden a la construcción de nuevos saberes.

Se identifican una serie de competencias docentes promotoras del aprendizaje significativo en los estudiantes a través del modelo de enseñanza e-learning. Entre ellas se destacan el conocimiento y utilización de las herramientas informáticas, conocimiento y utilización de técnicas de planificación y diseño en ambiente virtual; y conocimiento y utilización de métodos didácticos para e-learning y b-learning. El 
desarrollo de estas competencias en las instituciones educativas se dará en la medida que existan políticas de formación para que los docentes promuevan el diseño de actividades de enseñanza que estimulen el aprendizaje significativo en los estudiantes y se impulse el uso de las TIC (Imbernón, Silva, Guzman , 2011).

De otra parte se hace necesario implementar según Montes de Oca (2007)) "la alfabetización múltiple para los nuevos ambientes de aprendizaje".(p.13), destacando que los estudiantes deben desarrollar habilidades, conocimientos y aptitudes necesarias para acceder a la educación virtual y los docentes deben saber diseñar sus cursos en ambientes virtuales con metodologías y estrategias que garanticen una educación de calidad.

Es necesario motivar el desarrollo de competencias en los estudiantes en relación el nivel de conocimiento, habilidades y actitudes alcanzados en el uso del modelo de enseñanza e-learning. Frade (2009) afirma que: "Competencia es la capacidad adaptativa para desempeñarse frente a las demandas que se presentan en contextos diferenciados, con distintos niveles de complejidad. Es un pensar para poder hacer, ser y vivir en sociedad". (p.13).

Se han efectuado estudios que analizan el desarrollo de competencias genéricas y trasversales según las necesidades de formación de educación superior en Europa (Espacio Europeo de Enseñanza Superior EEES) con el objetivo de crear una metodología adaptada a la virtualidad y aplicada en el área de conocimiento de economía y empresa (Vilaseca, J., Meseguer, A., Ficapal, P., Torrent, J., Cortadas, P, 2006). También se han realizado estudios que miden las competencias que los tutores on line requieren para el desarrollo efectivo del modelo e-learninig (Yot, C.,Marcelo, C, 2013) otros, que analizan la incidencia de los componentes de interacción por medio de foros (Ehuleche, A., De Estefano, A.,2011), la influencia de la asesoría en el proceso de aprendizaje bajo ambientes virtuales (Pulford, 2009). En relación con el uso de la tecnología virtual se han elaborado estudios relacionados con la medición del desempeño en el manejo de las TIC, describiendo el perfil diferenciado de los usuarios (Ferran, N., Pérez, M., 2009) y la medición de experiencias y opiniones de aprendices virtuales en relación con los trabajos, los desafíos y las competencias que aseguran que el aprendizaje virtual sea exitoso (Beaudoin, M. Kurtz, G. Sigal, E, 2009) entre otros. No se ha efectuado un análisis hasta la fecha del desarrollo de competencias genéricas o transversales en relación al modelo e-learning en Colombia, tampoco se ha estudiado la relación del desarrollo de estas competencias con las destrezas en el manejo del modelo.

La sociedad actual demanda profesionales que estén capacitados para afrontar los diferentes contextos y problemáticas actuales, para ello es necesario formar para el desarrollo de competencias disciplinares relacionadas con el campo de conocimiento y en competencias genéricas que son aquellas que todo individuo debe haber desarrollado al finalizar un determinado plan de estudios (Frade, 2009). Es importante tener en 
cuenta el desarrollo de las competencias transversales que son aquellas competencias genéricas comunes a la mayoría de profesiones. En la tabla 1 La Red Mutis ${ }^{1}$ se determina y clasifica las competencias transversales para el desarrollo de los programas de pregrado de las instituciones que la conforman.

\section{Tabla 1}

\section{Competencias transversales Red Mutis}

\begin{tabular}{ll}
\hline Cognitivas & $\begin{array}{l}\text { Acción de aprender el conociendo necesario para la comprensión de la } \\
\text { fenomenología. La manera como el hombre se apropia de su lengua y la utiliza } \\
\text { para comunicarse con los demás. Capacidad que tienen los seres humanos para }\end{array}$ \\
\hline Socio afectivas & $\begin{array}{l}\text { Acción de interactuar con la realidad y sus agentes sobre la plataforma de } \\
\text { criterios axiológicos }\end{array}$
\end{tabular}

Comunicativas Acción de entendimiento y la aplicación de mecanismos para el trabajo en equipo

Relacionamiento Acción de relacionar la interdisciplinariedad inmersa en todo el fenómeno organizacional, con el fin de encontrar la solución más conveniente

Fuente: Red Mutis Uniminuto 2009

El propósito de esta red es consolidar cada una de las instituciones que la conforman y fortalecer la educación superior mejorando la calidad académica. También existen más redes universitarias que desarrollan el mismo propósito. Entre ellas está la Red Marista, ${ }^{2}$ AUI Asociación Internacional de Universidades, IESALC Instituto Internacional de la Unesco para la Educación Superior en América Latina y el Caribe entre otras.

Los programas de pregrado de las instituciones de educación superior en Colombia tienen la responsabilidad de estar diseñados por competencias de acuerdo con los parámetros de calidad exigidos por las mismas instituciones de educación superior y el Ministerio de Educación Nacional. Actualmente las instituciones de educación superior en Colombia ofertan programas universitarios de pregrado presenciales; pero muchas instituciones han ampliado su cobertura por medio del ofrecimiento de programas de pregrado a distancia tradicional que utilizan modelos de enseñanza-aprendizaje $b$ learning y e-learning, creando ambientes virtuales de enseñanza y aprendizaje que utilizan plataformas tecnológicas (Moodle, Blackboard) que facilitan la interacción y el desarrollo del conocimiento. Se diseñan así cursos que utilizan según el modelo de

\footnotetext{
${ }^{1}$ La Red Mutis está conformada por nueve instituciones universitarias: Instituto Tecnológico y de Estudios Superiores de Monterrey (ITESM), Corporación Universitaria Minuto de Dios, Universidad Tecnológica de Bolívar (UTB), Universidad Autónoma de Occidente (UAO), Universidad Autónoma de Bucaramanga (UNAB), Corporación Universitaria de Ibagué, Universidad Autónoma de Manizales, Fundación Universitaria de Popayán y la Fundación Suramericana de Medellín www.redmutis.org

2 La Red Marista Internacional de Instituciones de Educación Superior, es un tejido de obras de la Misión Compartida de hermanos y laicos que operan en diferentes Provincias del Instituto Marista htttp://www.champagnat.org/edu/000.php?p=269
} 
enseñanza-aprendizaje una proporción o la totalidad de virtualidad. En ambos casos es necesario el cumplimiento de los parámetros de calidad para el desarrollo de competencias trasversales en los estudiantes. Teniendo en cuenta estas situaciones es necesario preguntarnos ¿cómo el modelo e-learning, a partir de ambientes virtuales de aprendizaje, aporta al desarrollo de competencias trasversales, específicamente las competencias cognitivas, socio afectivas y comunicativas en los estudiantes en pregrado de programas a distancia? y, ¿cuál es el nivel de experticia que tienen los estudiantes en uso del modelo e-learning? Este es el objeto de estudio de la investigación realizada que contribuye a generar resultados que evidencian el éxito o el fracaso del modelo e-learning en relación con el desarrollo de las siguientes competencias transversales: competencias cognitivas, socio afectivas y comunicativas, determinando factores claves de éxito, descubriendo debilidades y proponiendo acciones de mejoramiento en la implementación del modelo para futuras aplicaciones.

El método de investigación empleado en el estudio fue mixto: cualitativo y cuantitativo, según Hernández Sampieri (2010) este método utiliza "las fortalezas de ambos tipos de indagación combinándolas y tratando de minimizar sus debilidades potenciales".(p.544). Se utilizó el método cualitativo para determinar el desempeño de los estudiantes en el desarrollo según la Red Mutis de competencias transversales cognitivas, socio afectivas y comunicativas en el modelo e-learning. Se observaron y analizaron las actitudes, comportamientos y motivaciones que tienen los estudiantes con el uso del modelo e-learning. El método utilizado para el desarrollo de este enfoque fue la investigación acción que busca el estudio y la reflexión sobre una situación o problemática, con el fin de proponer mejoras y cambiar el fenómeno (Giroux y Tremblay, 2008).

Se utilizó el método de investigación cuantitativo para determinar el nivel de desempeño del modelo con relación a la habilidad en el manejo de la información, conocimiento de los medios de interacción, conceptos informáticos, habilidades técnicas y capacidades intelectuales de orden superior, el método utilizado para el desarrollo de este enfoque fue el método de encuesta, seleccionando el cuestionario como técnica de investigación en la aplicación como instrumento en la muestra seleccionada por medio de un sondeo (Giroux y Tremblay, 2008).

\section{Metodología}

El escenario de desarrollo de esta investigación se sitúo en Villavicencio - Colombia, en el programa Administración de Empresas de la Corporación Universitaria Minuto de Dios. El tamaño de la población del estudio fue 145 estudiantes, distribuidos según la tabla 2 en tres grupos; dos eran del inicio del programa (primero y segundo semestre) y un grupo estaba situado al final del programa (noveno semestre). Los grupos del inicio del programa tenían un nivel básico de desarrollo de conocimientos de español y sistemas comparados con el grupo final que paso por la mayor parte del plan académico del 
programa. Los tres grupos cumplían con las condiciones para el estudio debido a que estos cursos son los únicos ofrecidos en el programa de forma virtual. El estudio se realizó en el mes de septiembre y octubre de 2012.

Tabla 2

Cursos y cantidad de estudiantes

\begin{tabular}{|c|c|c|}
\hline Curso/semestre & $\begin{array}{c}\text { Cantidad de estudiantes } \\
\text { primer año }\end{array}$ & Participación \\
\hline Proyecto de vida/Primer semestre & 90 & $62 \%$ \\
\hline Cátedra Minuto de Dios/Segundo semestre & 45 & $31 \%$ \\
\hline Simulación Gerencial/ Noveno semestre & 10 & $7 \%$ \\
\hline Total Población (N) & 145 & $100 \%$ \\
\hline
\end{tabular}

Se determinó para el estudio cualitativo tomar una muestra intencional de 15 estudiantes para realizar el estudio. Fueron seleccionados de forma aleatoria según listas de clase. La distribución para la selección se elaboró de acuerdo con la participación de cada grupo sobre el tamaño total de la población de esta forma: Proyecto de vida (8), Cátedra Minuto de Dios (4) y Simulación gerencial (3). La técnica de recolección de datos para este estudio fue entrevista en profundidad y la rejilla de observación.

El tamaño de la muestra para el estudio cuantitativo fue 105 estudiantes, grupo determinado de forma estadística con un intervalo de confianza del $95 \%$ y distribuidos así: Proyecto de vida (65), Cátedra Minuto de Dios (33) y Simulación gerencial (7). Fueron seleccionados de forma aleatoria según listas de clase. La técnica de recolección de datos para este estudio fue por medio de la utilización de encuesta realizada electrónicamente por internet.

La captura de los datos y análisis que se empleó para el enfoque cualitativo fue por medio de la identificación de índices emergentes y las categorías. Se validaron los resultados mediante el procedimiento de triangulación tomando la información de las entrevistas, las observaciones y algunos datos del estudio cuantitativo. Para el análisis del estudio cuantitativo se desarrolló mediante un manual de codificación según el planteamiento propuesto Giroux y Tremblay (2008) y la utilización del programa estadístico Spss.

\section{Resultados}

Los siguientes son los resultados de la aplicación de la técnica de recolección de datos rejilla de observación y entrevistas en profundidad realizadas para el estudio cualitativo en relación con la evaluación del desarrollo competencias transversales (competencias cognitivas, socio afectivas y de comunicación). 
Se analizaron las siguientes variables en el desarrollo de competencias cognitivas: procesamiento de la información, motivación reconceptualizada, mediación y metacognición.

En el procesamiento de la información todos los estudiantes seleccionados en el estudio respondieron como positiva la experiencia en relación a la comprensión de los conceptos vistos en los cursos virtuales, la mayoría de ellos estuvieron de acuerdo en que la atención debe ser total para trabajar en el aula virtual. Todos los entrevistados respondieron que no es necesario el uso de la memoria para aprender los contenidos de los cursos de las aulas virtuales.

En relación con la variable motivación reconceptualizada se encontró que la totalidad de los estudiantes valoraron los conceptos vistos en los cursos y los relacionaron con su crecimiento personal, todos le dieron una alta significación a los temas aprendidos demostrando percepción y comprensión de las temáticas y la puesta en práctica mediante el desarrollo de habilidades y destrezas.

En los procesos de mediación, manifestaron que es positivo el apoyo del docente en su proceso de aprendizaje valorando la retroalimentación obtenida. En el desarrollo del proceso de metacognición todos los seleccionados alcanzaron los logros en el proceso de aprendizaje relacionado con las metas propuestas.

En relación con el desarrollo de competencias socio afectivas se analizaron las siguientes variables: intensidad y duración de las sensaciones e interacción con la realidad y sus agentes son criterios axiológicos.

En Intensidad y duración de las sensaciones, en algunos casos no existió en el manejo de los cursos una relación de confianza y afecto con el docente, existió poco diálogo debido a la poca interacción en los foros del aula, para la mayoría de los estudiantes la mayor sensación que se dio fue el respeto y la armonía en la comunicación con sus compañeros. Todos estuvieron de acuerdo en que en la virtualidad puede haber interacción social, manifestando en términos generales motivación en el desarrollo del curso, aunque algunos sintieron ciertas frustraciones en relación a la interacción, las lecturas de las actividades y los problemas técnicos.

En la interacción con la realidad y sus agentes con criterios axiológicos, todos los estudiantes estuvieron de acuerdo en manifestar que los valores éticos en la virtualidad se fortalecen, pero algunos manifestaron que aunque se fortalezca, existen casos donde se pierden. 
En la tercera categoría de análisis competencias comunicativas se analizaron las siguientes variables: competencia lingüística, competencia comunicativa y competencia interactiva.

En el análisis de la competencia lingüística, todos los estudiantes estuvieron de acuerdo en manifestar que el lenguaje escrito que empleó el docente fue claro y asertivo. Algunos de ellos se quedaron callados cuando el lenguaje empleado en el aula no era de fácil comprensión.

En la competencia comunicativa se evidencia que los aportes y comentarios fueron oportunos, aunque unos pocos manifestaron que fueron tardíos. Algunos estudiantes tuvieron deficiencias en la utilización del lenguaje escrito, estas deficiencias se dieron principalmente en redacción, ortografía y en identificación de ideas; en general manifestaron tener facilidad para utilizar el lenguaje escrito, la mayor dificultad que han tenido en la comunicación es la dificultad técnica debido a cierres de la plataforma, comunicación vía internet, inicio de actividades clave, entre otros.

En la competencia interactiva, los estudiantes en su mayoría no tuvieron problemas para ponerse de acuerdo con sus compañeros y docente; en algunos casos se presentó poco entendimiento con el docente. Todos respetaron la opinión de los demás así no la compartieran en las aulas virtuales y manifestaron que cuando se presentaron controversias se llegó a un punto de acuerdo.

Los siguientes son los resultados de la aplicación de la técnica de recolección de datos: encuesta para el estudio cuantitativo en relación a la evaluación del desarrollo de competencias en destrezas en el manejo del modelo e-learning llamado Experticia según Eduteka (2007). Se analizó el nivel presentado y su uso. En la figura 1 se presenta el resultado del promedio de los resultados de los siguientes indicadores: conceptos informáticos fundamentales, habilidades técnicas en el uso de herramientas y capacidades intelectuales de orden superior de esta categoría. 


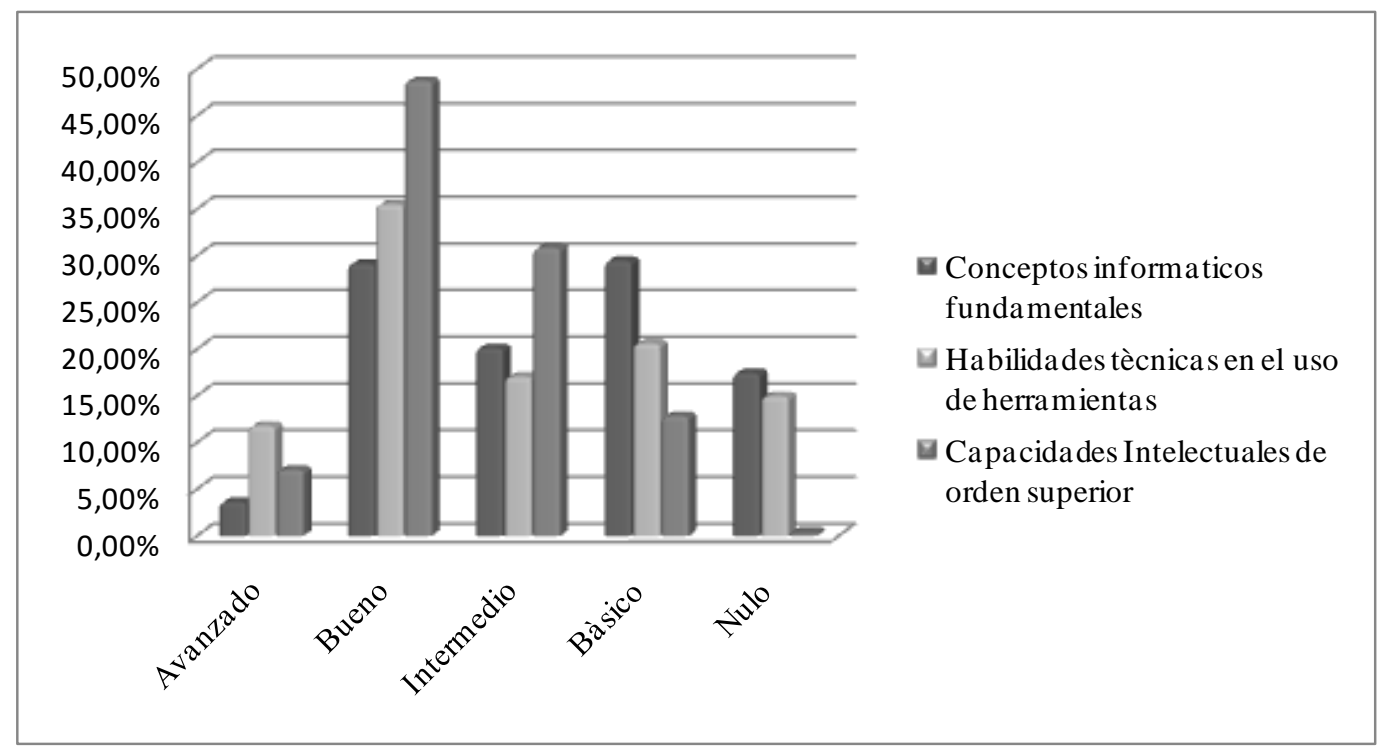

Figura 1. Nivel de Experticia Conceptos informáticos fundamentales, habilidades técnicas en el uso de herramientas y capacidades intelectuales de orden superior

Fuente: datos recabados por el autor

En relación con la búsqueda de la información los estudiantes del estudio demostraron tener buenas capacidades para seleccionar entre múltiples hallazgos el mejor resultado de una búsqueda de diversas fuentes como libros guía y de consulta. Se evidenció interés por investigar datos en la web de forma confiable para sus trabajos. Estas habilidades fortalecen las destrezas de los estudiantes para la toma de decisiones en diferentes escenarios porque crea la necesidad de buscar información confiable para este propósito. En la figura 2 se presenta el resultado del promedio del indicador búsqueda de información.

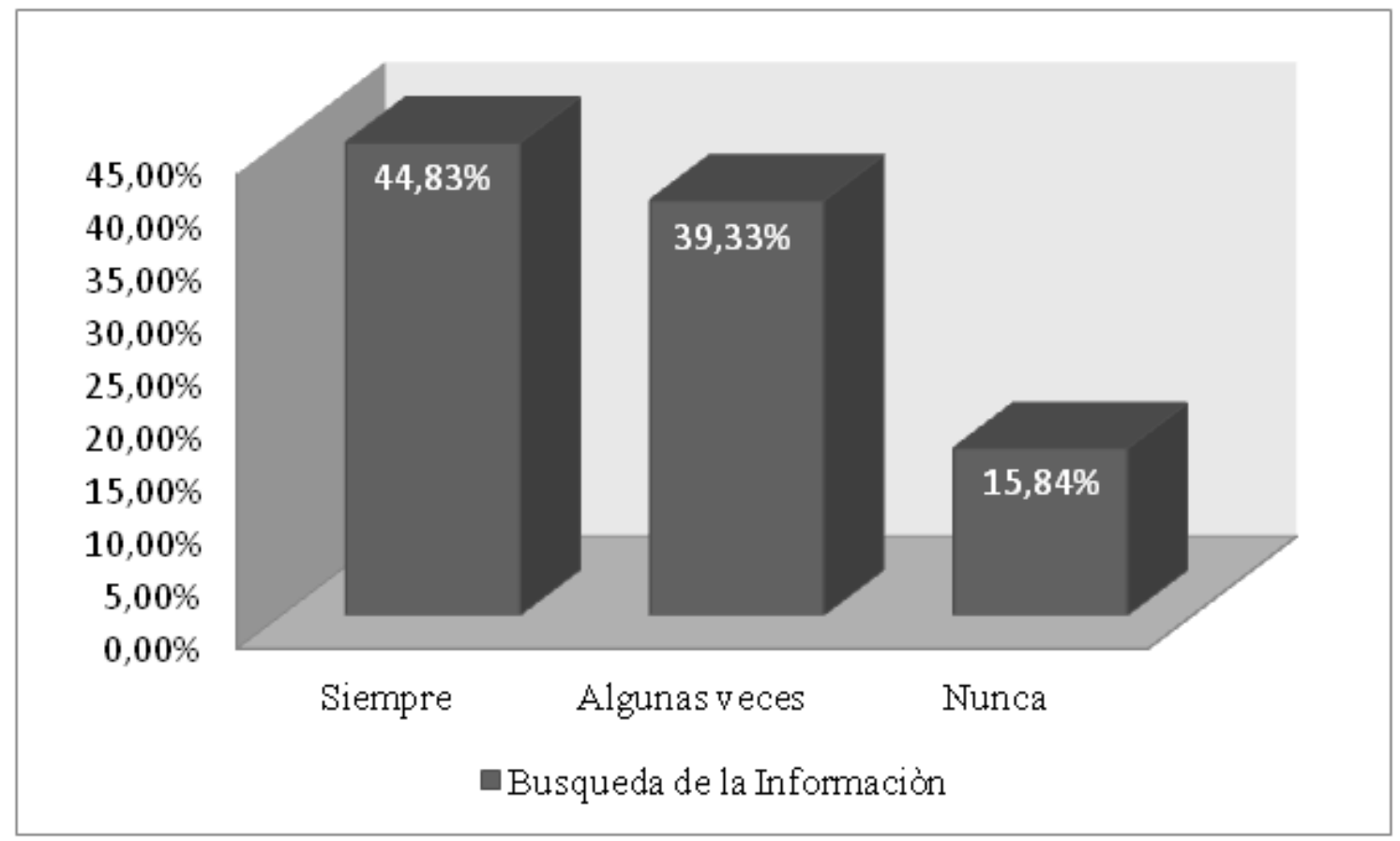

Figura 2. Nivel de Experticia para la búsqueda de Información

Fuente: datos recabados por el autor

Evaluación del desarrollo de competencias transversales y destrezas en el manejo del modelo e-learning en programas de pregrado en Latinoamérica. Juan Fernando Ossa Stipcianos.

Página 11 de 28 
En los conceptos informáticos fundamentales los estudiantes del estudio obtuvieron un buen nivel en conocimientos básicos de los conceptos de informática y algunos demostraron que manejan de forma adecuada la representación digital (elaboración de imágenes virtuales). Se evidenció un manejo adecuado de la plataforma de información de la Universidad, aunque tienen un nivel bajo en el manejo de modelos (elaboración de software) y en programación. Se puede determinar que el nivel de conocimiento de los conceptos de informática está entre el nivel básico e intermedio.

En las habilidades técnicas en el uso de herramientas no hay un conocimiento amplio en el manejo técnico del hardware e instalación de computadores. Los estudiantes demostraron conocimiento en el manejo de correo y comunicaciones, navegador, hoja de cálculo Excel, procesador de texto Word y presentador multimedio Windows media player. Presentaron un nivel medio y bajo en el manejo de imágenes digitales, bases de datos, instalación de computadores, uso de funciones básicas y sistemas operativos. En este aspecto se puede afirmar que los estudiantes se ubican en el nivel intermedio.

En las capacidades intelectuales, las más altas son en orden de mayor a menor: manejo de la complejidad, investigación, solución de problemas, toma de decisiones, creatividad, pensamiento crítico, y prueba de soluciones, Las capacidades más bajas de los estudiantes son el razonamiento sostenido e innovación. En este aspecto las capacidades intelectuales se encuentran entre el nivel intermedio y bueno.

En relación al uso del Modelo e-learning los resultados académicos en el uso del modelo e-learning de de los cursos evaluados en el estudio son favorables, la mayoría de los estudiantes demuestran aprobar las materias virtuales en buen nivel logrando obtener una motivación intrínseca en el logro de sus resultados. Según el estudio el aprendizaje se da en mayor proporción por medio del entendimiento de forma autónoma atendiendo y aplicando las instrucciones y los conceptos del curso virtual. De igual manera, investigan y comprenden los contenidos definidos previamente por el docente. Buscan con frecuencia la explicación de otro compañero y la retroalimentación del docente. La mitad de los estudiantes utiliza un lenguaje comprensible que incluye el uso de abreviaturas en los foros y el chat, pero algunos de los estudiantes tienen problemas en el entendimiento del significado de los mensajes.

\section{Discusión}

Se evidencia el desarrollo de competencias transversales cognitivas, socio afectivas y comunicativas en el desarrollo de los cursos analizados (Proyecto de Vida, Cátedra Minuto de Dios y Simulación Gerencial) bajo la modalidad de enseñanza e-learning del programa Administración de Empresas en Uniminuto.

Para el desarrollo de las competencias cognitivas es necesaria la comunicación 
permanente del docente con los estudiantes mediante un proceso adecuado de mediación en las aulas virtuales y el desarrollando experiencias de aprendizaje. Según Lozano (2002) experiencias de aprendizaje es: "toda actividad planeada, dirigida y evaluada por el profesor o el departamento académico, para que el alumno desarrolle una serie de conocimientos, habilidades y actitudes".(p.104), es necesario un alto compromiso del docente en el desarrollo en el aula de conceptos declarativos (relacionados con la materia), procedimentales (relacionados con habilidades) y actitudinales (relacionados con actitudes) en los estudiantes. Para que sea efectivo este proceso es necesario aplicar eficientes métodos de comunicación escrita en diferentes medios (chat, foros, correo electrónico) de forma permanente, clara, concisa y amable. Adicionalmente, desarrollar un proceso social para el aprendizaje en los estudiantes mediante el apoyo y la colaboración en la comprensión de los contenidos, utilizando el lenguaje como medio regulador. Un buen proceso de comunicación logra en el estudiante obtener un aprendizaje significativo según Ausubel (1963) para relacionar los conceptos nuevos con la información y los modelos mentales existentes, cambiando en él la forma de pensar, de actuar y de entender; se establece en el estudiante un interés de forma intrínseca en las temáticas y las actividades de cada curso.

Es importante el desarrollo de motivación intrínseca acompañada del desarrollo de estrategias de comunicación efectiva por parte del docente para fortalecer compromiso de los estudiantes. Es importante que el estudiante haya internalizado principios bien determinados que garantizan el desarrollo de competencias socio afectivas.

Los resultados del estudio relacionados con el nivel de experticia demuestran que existe interés en el estudiante de buscar la información necesaria en la web y en otros medios para el logro de los resultados en su proceso de aprendizaje con la utilización del modelo e-learning. Los resultados del estudio evidenciaron que los estudiantes tuvieron un adecuado manejo de la información, seleccionando el mejor resultado en su búsqueda e investigado en otros medios como libros impresos de consulta y libros guía. Demostraron un comportamiento ético al buscar la información por sus propios medios para sus investigaciones. Para Castells (1997, p. 83) citado por García (2007), la sociedad de la información no depende del incremento de la productividad sino de la aplicación de conocimientos e información a la gestión, producción y distribución, tanto en los procesos como en los productos. De acuerdo con esto, las habilidades en la búsqueda de información garantizan el desarrollo de destrezas en los estudiantes para la toma de decisiones en diferentes escenarios.

Se pudo evidenciar que existe conocimiento de los conceptos informáticos fundamentales. Los estudiantes del estudio demostraron que tienen un buen nivel en conocimientos básicos de informática y algunos manejan de forma adecuada la representación digital (elaboración de imágenes virtuales), emplean adecuadamente la plataforma de información de la Universidad pero tienen un nivel bajo en el manejo de Modelos (elaboración de software) y en programación. El hombre es un ser social 
que necesita comunicarse y relacionarse con los demás, las TIC ayudan a este proceso por medio innovadoras formas de asociación llamadas sociedades virtuales o comunidades virtuales (Rabiela, 2009). La interacción exitosa de los estudiantes en las comunidades virtuales depende del dominio en conocimientos informáticos de cada participante.

Los resultados evidencian que las habilidades técnicas más utilizadas de herramientas informáticas son las de naturaleza básica, no hay un conocimiento amplio en el manejo técnico del hardware. Los estudiantes demuestran conocimiento en el manejo de correo y comunicaciones, navegador, hoja de cálculo Excel, procesador de texto Word y Windows Media Player. Presentan un nivel medio y bajo en el manejo de imágenes digitales, bases de datos, instalación de computadores, uso de funciones básicas y sistemas operativos. Para García (2012) a través de la información surge el conocimiento una vez que se le ha dado significado a los datos, con apoyo de la educación, experiencias previas y valores. Para poder utilizar la información y crear conocimiento en las aulas virtuales es necesario dominar todas las habilidades técnicas de las herramientas informáticas.

Las capacidades de orden superior más altas de los estudiantes son en orden de mayor a menor: manejo de la complejidad, toma de decisiones, investigación, solución de problemas, creatividad, prueba de soluciones y razonamiento sostenido. Las capacidades más bajas de los estudiantes son la innovación y el pensamiento crítico. Según Eduteka (2009) la experticia tiene relación con una serie de competencias en el manejo de la información y en la construcción del conocimiento. La generación de conocimiento por medio de las TIC depende del dominio todas las capacidades intelectuales de orden superior; la calidad de la construcción de este conocimiento dependerá del nivel de desarrollo de cada una de ellas.

\section{Conclusiones}

Es importante en los cursos bajo modalidad e-learning la comunicación y la interacción entre los estudiantes y docentes. El hombre es un ser social que necesita comunicarse y relacionarse con los demás las TIC ayudan a realizarlo por medio de innovadoras formas de asociación llamadas sociedades virtuales o comunidades virtuales (Rabiela, 2009). Los cursos en modalidad e-learning ayudan estimular el desarrollo de estas comunidades de aprendizaje y la interacción en entornos distintos al aula de clase, utilizando nuevas formas de relacionarse en escenarios y canales nuevos de comunicación sincrónica y asincrónica llamados Ambientes Virtuales de Aprendizaje (Balderas, 2009).

Es fundamental desarrollar procesos de metacognición en los estudiantes entendiendo por metacognición según Flavell (1987) citado por Sandoval, A., Franchi, L. (2007) como la capacidad para regular en el estudiante su propio aprendizaje, planificando 
estrategias para utilizar en cada situación, aplicar y controlar el proceso, evaluar posibles fallos y consecuencias, generar consciencia en los resultados y logro del aprendizaje. En el estudio, los estudiantes alcanzaron los logros en el proceso de aprendizaje relacionado con las metas propuestas. En 1960, J. Bruner expuso que el individuo tiene la tendencia a aprender sobre el ambiente, a innovar, a tener iniciativa y responsabilidad individual. Este argumento demuestra que los estudiantes deben desarrollar responsabilidades y compromisos con su aprendizaje de forma autónoma para el desarrollo de competencias cognitivas en cursos virtuales de aprendizaje.

Para que exista un buen desarrollo de competencias cognitivas en los cursos de modalidad e-learning es necesario que exista permanentemente la guía del Tutor. Los resultados del estudio, demostraron que los estudiantes valoran de forma positiva el apoyo del Tutor en su proceso de aprendizaje y la retroalimentación oportuna de su ejercicio académico. En 1920, Vigotsky aseguró que es el lenguaje el que enlaza cerebro y conducta. Según esto es necesaria la comunicación permanente del tutor con sus estudiantes para garantizar un efectivo desarrollo de competencias cognitivas y de mediación en las aulas virtuales.

Es necesario que el docente incluya en el diseño instruccional ${ }^{3}$ escogido de acuerdo al análisis del contexto y las necesidades de aprendizaje una serie de actividades o experiencias significativas que propicien el trabajo en equipo, el aprendizaje colaborativo y la comunicación dialógica, incluyendo actividades que fomenten la participación de los estudiantes en grupos para la solución de problemáticas y el desarrollo de conceptos. Es importante que se apliquen modelos y estrategias de enseñanza como: aprendizaje basado en problemas, debate y argumentación que garanticen la participación activa del estudiante en su aprendizaje.

Para lograr este aspecto es importante que los docentes tengan formación en educación y pedagogía para la aplicación de estas estrategias. Se debe desarrollar competencias en empatía, comunicación, habilidad para escribir y entendimiento de mensajes; este ejercicio permite que los estudiantes se involucren con las temáticas. Un docente que aplique las competencias mencionadas podrá ser un verdadero asesor, al poner en acción habilidades de interpretación, de empatía positiva y habilidades para retroalimentar el aprendizaje (Lozano, 2005).

\footnotetext{
${ }^{3}$ Diseño instruccional es el desarrollo sistemático de los elementos instruccionales, usando las teorías del aprendizaje y las teorías instruccionales para asegurar la calidad de la instrucción. Incluye el análisis de necesidades de aprendizaje, las metas y el desarrollo materiales y actividades instruccionales, evaluación del aprendizaje y seguimiento (Berger y Kam, 1996). Existen varios modelos de diseños instruccionales entre ellos está el modelo de Gagnè y Briggs de naturaleza cognitivista en el que propone un enfoque de sistemas de 14 pasos estructurado en cuatro niveles de operaciones sistémicas que facilitan la elaboración y el desarrollo de la instrucción, es utilizado en los planes de curriculares de sistemas educativos (Nieto, 2009). También está el modelo de Dick, Carey y Carey (2005) de naturaleza conductivista, es un sistema compuesto de 10 pasos consecutivos que conlleva al aprendizaje autónomo, es un diseño sistemático que se somete a revisión y a una evaluación permanente.
}

Evaluación del desarrollo de competencias transversales y destrezas en el manejo del modelo e-learning en programas de pregrado en Latinoamérica. Juan Fernando Ossa Stipcianos.

Página 15 de 28 
El desarrollo de competencias transversales cognitivas, socio afectivas y comunicativas depende en gran medida de las destrezas que tengan los estudiantes en el manejo y desarrollo de su aprendizaje por medio del modelo de enseñanza e-learning, como también depende del apoyo del docente en el diseño, desarrollo de cada actividad y del seguimiento del desempeño cada uno de los estudiantes.

Uno de las mayores limitaciones para efectuar este estudio fue la disponibilidad de los estudiantes para dar la información cuando era necesaria y la disposición para hacerlo debido a sus compromisos académicos. Para estudios futuros es necesario evaluar la forma como los docentes están utilizando el modelo e-learning midiendo su nivel de destreza en Tics y la evaluación del aprendizaje por competencias.

Las implicaciones de este estudio serán evidentes en el desarrollo del mejoramiento del desempeño de los estudiantes y los docentes de los cursos bajo modalidad e-learning buscando mejorar la calidad académica de los programas. En los estudiantes implica obtener un desempeño óptimo en competencias, logrando resultados en conocimientos adquiridos y habilidades en la puesta en práctica de esos conocimientos, con actitudes como responsabilidad en su aprendizaje autónomo ${ }^{4}$, respeto hacia los demás, colaboración y trabajo en equipo. En los docentes se requiere mejorar la práctica docente mediante el mejoramiento en el diseño de los cursos en contenidos y actividades que logren obtener en el estudiante experiencias significativas de aprendizaje. Es necesario capacitar a los docentes en modelos y estrategias de enseñanza y en mecanismos de comunicación efectiva que den elementos para mejorar el diseño y la gestión de ambientes virtuales de aprendizaje.

Presentación del artículo: 15 de febrero de 2016

Fecha de aprobación: 4 de marzo de 2016

Fecha de publicación: 30 de abril de 2016

Ossa, J. (2016). Evaluación del desarrollo de competencias transversales y destrezas en el manejo del modelo e-learning en programas de pregrado en Latinoamérica. RED. Revista de Educación a Distancia. 49(14). Consultado el (dd/mm/aaaa) en http://www.um.es/ead/red/49

\footnotetext{
${ }^{4}$ Aprendizaje autónomo es el cumplimiento de logros a través de interacciones organismo-objetivo, se concibe en términos de su independencia respecto al número y variedad de elementos ajenos al propio aprendiz, complementada con dependencia a actividades del sujeto (Moreno, R, Martínez, J, 2007).
} 


\section{Referencias bibliográficas}

Ausubel, D. (1963). The psychology of meaningful verbal learning. New York, Grune and Stratton

Alvarado, M., y Calderón, I. (2013). Diagnóstico Estadístico y Tendencias de la educación superior a distancia en Colombia . En Educación superior a distancia virtual en Colombia: Nuevas Realidades. Bogotá, Colombia: Asesad/Virtual Educa.

Americalearningmedia.com.(2016). Tendencias para el e-learning en 2016. Retrieved 21 January 2016, Recuperado el 10 de enero de 2016 de: http://www.americalearningmedia.com/edicion-043/483-tendencias/6941-tendenciaspara-el-e-learning-en-2016

Anton, J., Rincón Prado, J. (2013). La educación superior a distancia virtual en Colombia: Nuevas Realidades. Bogotá, Colombia: Asesad/Virtual Educa.

Balderas, R.(2009). ¿Sociedad de la información o sociedad del conocimiento? [Versión electrónica].Revista de la Realidad Mexicana 158, 75-80

Beaudoin, M. Kurtz, G. Sigal, E. (2009). Experiences and Opinions of E-learners: What Works, What are the Challenges, and What Competencies Ensure Successful Online Learning. [Versión electrónica]. Interdisciplinary Journal of E-Learning and Learning Objects.

Berger, C. \& Kam, R. (1996). Definitions of Instructional Design. Adapted from "Training and Instructional Design". Applied Research Laboratory, Penn State University. Recuperado el 13 de marzo de 2016, de: http://www.umich.edu/ ed626/define.html

Bruner, J. S. (1960). The Process of education. Cambridge, Mass.: Harvard University Press.

Castell, M. (1997). La era de la información. Economía, sociedad y cultura. Volumen 1. La sociedad red. Alianza Editorial, Madrid

Dick, W., Carey, L., Carey, J. (2005). The systematic design of instruction, (6th ed.). USA: Person.

Echeverría, J. (1999). Los señores del aire: telépolis y el tercer entorno. Destino. Barcelona.

Eduteka Portal Educativo gratuito de la Fundación Gabriel Piedrahita Uribe. (2007). El porqué de las TIC en educación. Recuperado el 21 de agosto de 2011 de: http://www.eduteka.org/PorQueTIC.php

Eduteka Portal Educativo gratuito de la Fundación Gabriel Piedrahita Uribe. (2009). Impacto de las TIC en escuelas Europeas. Recuperado el 21 de agosto de 2011 de: http://www.eduteka.org/ICT_InformeEuropa.php 
Ehuleche, A., De Estefano, A. (2011). Evaluación de la competencias para la formación tutores de e-learning. [Versión electrónica]. Revista Ried. 14 (1)

Estévez, J. A., Castro-Martínez, J., \& Granobles, H. R. (2015). La educación virtual en Colombia: exposición de modelos de deserción [1]. (Spanish). Apertura: Revista De Innovación Educativa, 7(1), 1.

Facundo, A. (2002). La educación superior a distancia/virtual en Colombia. Recuperado el 10 de enero de 2016 de: http://unesdoc.unesco.org/images/0013/001399/139922s.pdf

Ferran, N, Pérez, M. (2009). Gestión de la información personal en usuarios avanzados en TIC. [Versión electrónica] El profesional de la información. 8(4) p. 365-37

Frade, Laura. (2009). Desarrollo de competencias en educación: desde preescolar hasta el bachillerato. México: Inteligencia educativa

Garcés Arguello, E. R., \& Rivera Enriquez, C. J. (2006). Evaluación de plataformas tecnológicas para la teleformación o e-learning para el ámbito universitario, tomando como caso de estudio educativa.

García, A. (2012). Sociedad del conocimiento y la educación. Madrid, España. Universidad Nacional de Educación a Distancia. Editor Lorenzo González Aretio.

García, A., Ruiz, M., Domínguez, D. (2007). De la educación a distancia a la educación virtual. Barcelona, España. Editorial Ariel S.A S. L.

Giroux, S., Tremblay, G. (2010). Metodología de las ciencias humanas. Fondo de cultura económica. México

Hernández, R., Fernández, C., Batista, M. (2010). Metodología de la Investigación. México. Editorial Mc Graw Hill

Iesalc (2006). Informe de la educación superior en América Latina (2000-2005). La metamorfosis de la educación superior en America Latina. Caracas: Iesalc.

Imbernón, Silva, Guzmán. (2011). Competencias en los procesos de enseñanza aprendizaje virtual y semipresencial. [Versión electrónica]. Revista Científica de Educomunicación. 36 (18) p 107-114

Lozano R, Armando. (2005). El éxito en la enseñanza. Aspectos didácticos de las facetas del profesor. México: Trillas.

Martínez Barrios, P. (2013). Prologo. En Arboleda, N., y Rama, C (2013). La educación superior a distancia virtual en Colombia: Nuevas Realidades. Bogotá, Colombia: Asesad/Virtual Educa.

Montes de Oca, R. (2007) Alfabetización múltiple en nuevos ambientes de aprendizaje. Villahermosa, Tabasco, México. Editorial Universidad. J. Autónoma de Tabasco. 
Moreno, R, Martínez, J. (2007). Aprendizaje autónomo. Desarrollo de una definición. [Versión electrónica]. Revista Latina de Análisis de Comportamiento. Revista Latina de Análisis de Comportamiento, 15 (1) p. 51- 62

Nieto, M. (2009). Tema 2 Parte I Diseño Instruccional, Teorías y Modelos Recuperado el 20 de enero de 2015 de: http://www.slideshare.net/mnieto2009/tema-2-parte-idiseo-instruccional-teoras-y-modelos\#

Obs-edu.com. (2014). La industria del e-learning duplicará su volumen hasta superar los 100.000 millones de dólares en 2015 - Actualidad. Recuperado el 21 January 2016 de: $\quad$ http://www.obs-edu.com/noticias/obs-business-school/la-industria-del-elearning-duplicara-su-volumen-hasta-superar-los-100-000-millones-de-dolares-en2015/

Pulford, B. (2009). The influence of advice in a virtual learning environment. [Versión electrónica]. British Journal of Educational Tecnology. 42 (1) p 31-39

Rabiela, M. (2009). Globalización de la educación. Comunidades virtuales de aprendizaje y su participación en las instituciones educativa.[Versión electrónica]. Hospitalidad-Esdai, 16(1), 37-60

Rama, C. (2013). El contexto de la reforma de la virtualización en América Latina. En Arboleda, N., y Rama, C (2013). La educación superior a distancia virtual en Colombia: Nuevas Realidades. Bogotá, Colombia: Asesad/Virtual Educa

Sandoval, A, Franchi, L. (2007). Meta comprensión en estudiantes de Ingeniería. [Versión electrónica]. Revista Omnia. 13 (2) p 98-119

Sarmento, M. Durão, D. (2009). Ethics dimensión in e-learning. [Versión electrónica]. Revista de Administração FACES Journal. 8(2) p 44-53

Suárez, E. J. C., Salinas, E. R (2009). Experiencias en e-Learning en Instituciones de Educación Superior en Colombia. Elizcom Sas.

Toffler, A. (2006). La revolución de la Riqueza. Bogotá: Debate.

Uniminuto (2009). Proyecto Curricular del Programa Administración de Empresas PCP. Bogotá, Colombia

Vigotsky, L.(1934). La psicología y la teoría de la localización de las funciones psíquicas (vol. 1). Madrid, Visor.

Vilaseca, J., Meseguer, A., Ficapal, P., Torrent, J., Cortadas, P. (2006). E-learning y desarrollo de competencias: la micronizaciòn de contenidos en economía y empresa. [Versión electrónica]. Revista Latinoamericana de Tecnología. 5 (2) p 113 - 147

Yot, C.,Marcelo, C, (2013). Tareas y competencias del tutor on line. [Versión electrónica]. Profesorado: Revista del Curriculum y formación del profesorado. 17 (2) 


\section{Anexo 1. Instrumento de recolección de la información Entrevista}

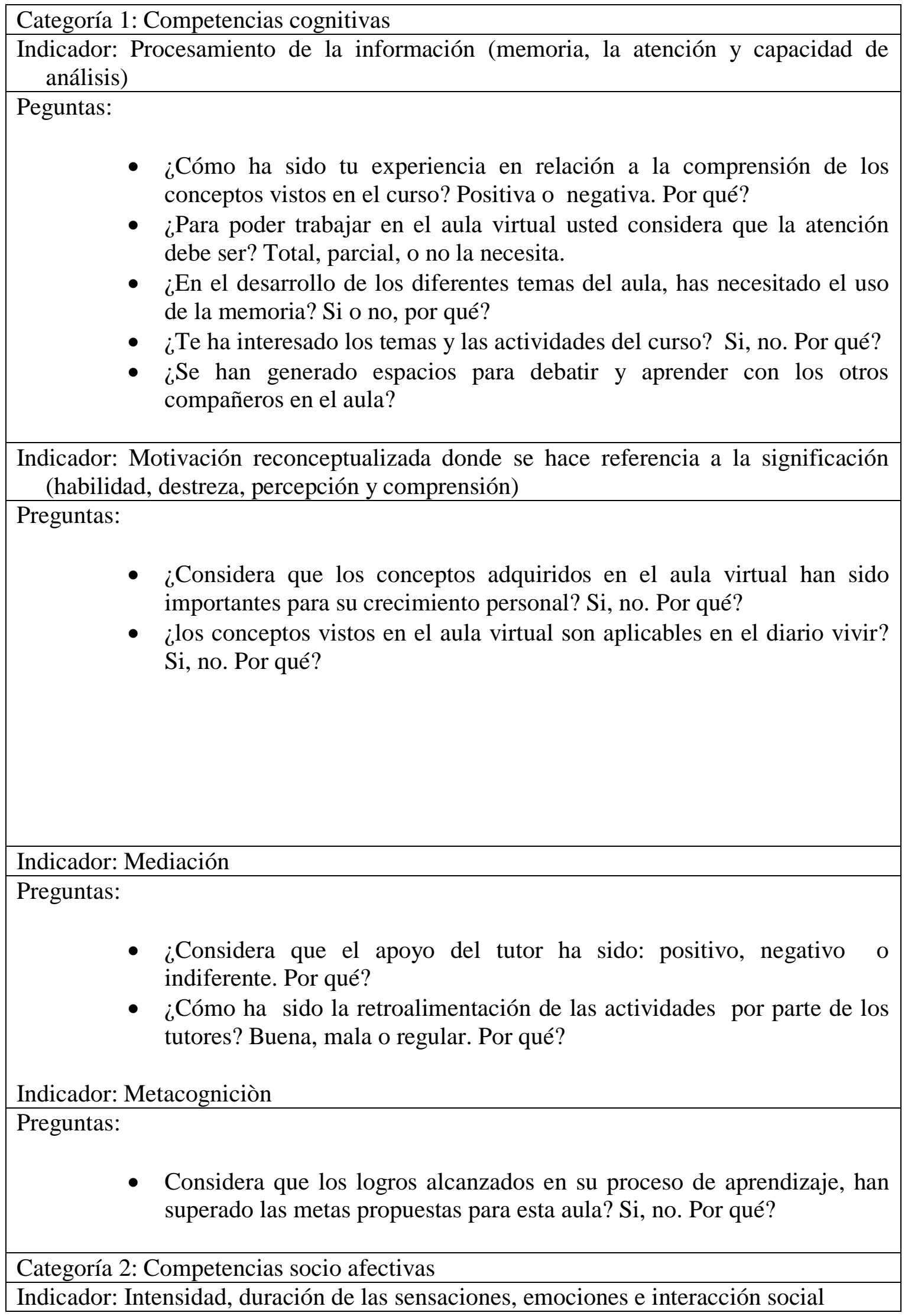




\section{Preguntas:}

- Consideras que has tenido una buena relación afectiva con tu tutor? Si, no. Por qué?

- Consideras que la relación con tus compañeros ha sido: buena, regular o mala?

- Ha existido en la comunicación con tus compañeros o tutor respeto, confianza, armonía, dialogo y entendimiento? Cual se ha dado en mayor y menor proporción?

- Consideras que con la virtualidad se puede dar la interacción social? Si, no. Por que?

- Como te has sentido en el curso? Frustrado, motivado, enojado u otra Por qué?

Indicador: Interacción con la realidad y sus agentes son criterios axiológicos

Preguntas:

- Consideras que los valores éticos en la virtualidad se: fortalecen, se pierden. Por qué?

- Tu interacción y compromiso cumplen con los reglamentos del curso y de la universidad? Si, no. Por que?

Categoría 3: Competencias comunicativas

Indicador: Competencia lingüística (lenguaje y actuación)

Preguntas:

- El lenguaje escrito utilizado por el tutor es claro y asertivo? Si, no. Por qué?

- Cuál es tu actitud cuando el lenguaje empleado en el aula, no es de fácil comprensión? Me quedo callado, solicito aclaración, tomo una actitud agresiva.

Indicador: Competencia comunicativa (cuando comunicarse y que decir)

Preguntas:

- Tus aportes y comentarios en las actividades en el aula han sido? Oportunas, tardías. Por qué?

- Al utilizar el lenguaje escrito tiene dificultades? Si, no. Por qué?

- Tienes facilidad para utilizar el lenguaje escrito? Si, no. Por qué?

- Al comunicarte en el aula virtual has tenido dificultades? Técnicas, comunicación o tiempo. 


\begin{tabular}{|l|}
\hline Indicador: Competencia interactiva (entendimiento con los demás) \\
\hline Preguntas: \\
- Se han presentado problemas para ponerse de acuerdo con los \\
compañeros y tutor? \\
Cuando tiene que realizar actividades en equipo establecen normas de \\
trabajo? Si, no. Por qué? \\
Cuando trabajamos en el aula respetamos la opinión de los demás asi no \\
la compartamos? Si, no. Por qué? \\
Cuando trabajamos en el aula y se presentan controversias, se llega a un \\
punto de acuerdo? Si, no. Por qué? \\
\end{tabular}




\section{Anexo 2. Instrumento de recolección de la información Encuesta}

\section{Anexo Encuesta}

Esta encuesta tiene como objetivo medir el manejo y el desempeño de los estudiantes en el desarrollo de los cursos virtuales utilizando la plataforma Moodle.

Nombre:

Sexo: Masculino

Femenino

Edad: Menor de 15 años

Entre 16 y 17 años _-

Entre 18 y 20 años

Entre 21 y 25 años

Mayor de 25 años

Semestre: Primero

Segundo

Noveno

Jornada: Diurno

Nocturno

Sabados

Curso visto en Moodle (Plataforma Virtual)

Proyecto de vida

Cátedra Minuto de Dios

Simulación Gerencial

1. ¿Cómo es tu manejo de la información en la web para el desarrollo una actividad del curso virtual? * 


$\begin{array}{rrr}\text { Algunas } & \text { Nunca } \\ \text { veces } & \end{array}$

Selecciono lo primero que aparezca en el buscador

Averiguo los resultados de varias opciones y escojo la más importante $\mathrm{y}$ pertinente.

Busco en otros medios diferentes a la Web como libros y lecturas el tema a investigar

Copio la información de otro compañero sin hacer el ejercicio investigativo

Utilizo las bases de datos (Ebsco, elibro ) de la biblioteca virtual Uniminuto

No me interesa buscar información y no presento los ejercicios

2. ¿Tu comprensión de los mensajes y las abreviaturas en el chat y en los foros es? ${ }^{*}$ Elige una opción

- $\quad$ Entendiendo todo el significado

- $\square$ Entendiendo parte del significado

- $\Gamma$ No entiende el significado

3. ¿Tu producción de mensajes y abreviaturas en el chat y en los foros es? $*$ Elige una opción

- $\Gamma$ Utilizo un lenguaje comprensible sin uso de abreviatura

- $\Gamma$ Utilizo un lenguaje comprensible con algunas abreviaturas

- $\square$ Utilizo solo términos y abreviaturas

4. ¿Cuál es tu nivel de conocimiento en los siguientes conceptos informáticos?*

Evaluación del desarrollo de competencias transversales y destrezas en el manejo del modelo e-learning en programas de pregrado en Latinoamérica. Juan Fernando Ossa Stipcianos.

Página 24 de 28 


\section{Nulo Básico Intermedio Bueno Avanzado}

Informática

(conocimientos

en herramientas)
Redes de
computadores
(Conexión de área local)

Sistemas de

información

(Sistema

Génesis)

\section{Representación}

digital

(Elaboración de imágenes

virtuales)

\section{Modelos}

(Elaboración de solfware)

Pensamiento

algorítmico

(Programación)

5. ¿Cuál es tu nivel de desempeño en el uso de herramientas informáticas? *

Nulo Básico Intermedio Bueno Avanzado

Instalación de computadores

Uso de funciones

básicas

(Comandos y

teclas alternas)

Sistema operativo

(Windows y

Linux)

Procesador de texto

(Word)

Presentador

multimedia

(Windows media 


\section{Nulo Básico Intermedio Bueno Avanzado}

$$
\text { player) }
$$

Navegador (Google)
Correo y
comunicaciones
(Yahoo, hotmail)

Hoja de cálculo (Excel

Bases de datos (Bibliotecas virtuales)

Imágenes digitales (Photoshop

6. ¿Cómo es tu desempeño en las siguientes capacidades generales? *

\section{Nulo Básico Intermedio Bueno Avanzado}

Creatividad

$\begin{array}{lr}\text { (Capacidad } & \text { de } \\ \text { producir } & \text { cosas } \\ \text { nuevas } & \mathrm{y} \\ \text { valiosas) } & \end{array}$

Innovación

(Capacidad de crear un producto nuevo o modificarlo)

\section{Investigación}

(Capacidad de obtener nuevos conocimientos)

Pensamiento crítico

(Capacidad de analizar evaluar

y




\section{Nulo Básico Intermedio Bueno Avanzado}

consistencia de

los

razonamientos)

Solución de

problemas

(Capacidad de buscar soluciones

a situaciones específicas)

Toma de decisiones

(Proceso en el cual se elige una opción o formas de resolver situaciones)

\section{Razonamiento}

sostenido

(Capacidad de extraer conclusiones de forma verídica)

Manejo de la complejidad

(Habilidad para alcanzar $\quad \mathrm{y}$ mantener un adecuado nivel de desempeño en tareas que son de nuestro interés)

Prueba de soluciones

(capacidad para poner y evaluar el desarrollo de una solución)

7. ¿Los conocimientos adquiridos en el curso han sido aprendidos de la siguiente forma? *

Siempre $\quad \begin{aligned} & \text { Algunas } \\ & \text { veces }\end{aligned}$

Mediante la investigación y la comprensión de los 


\section{$\begin{array}{rrr}\text { Algunas } & \text { Nunca }\end{array}$}

conocimientos con

objetivos definidos

previamente por el tutor

Mediante el uso de la memoria

Mediante la

retroalimentación del tutor

Mediante la explicación de otro compañero

Mediante el entendimiento

propio de las
instrucciones y los conceptos

8. ¿Mis resultados académicos en el curso virtual han sido? * Elige una sola opción
- $\Gamma$ Malo
- $\Gamma$ Regular
- Г Bueno
- $\square$ Muy bueno
- $\Gamma$ Excelente 